\title{
THE SUBPROJECTIVITY OF THE PROJECTIVE TENSOR PRODUCT OF TWO $C(K)$ SPACES WITH $|K|=\aleph_{0}$
}

\author{
ELÓI MEDINA GALEGO AND CHRISTIAN SAMUEL
}

(Communicated by Thomas Schlumprecht)

\begin{abstract}
We prove that the projective tensor product of two $C(K)$ spaces, where $K$ is an infinite countable metric compact space, is $c_{0}$-saturated and is therefore a subprojective space. This completes some recent work on subprojectivity of projective tensor products involving $C(K)$ spaces by T. Oikhberg and E. Spinu.
\end{abstract}

\section{INTRODUCTION AND THE MAIN THEOREM}

We use standard terminology and notation on Banach space theory; see e.g. 44. Subspace means a closed linear infinite dimensional subspace. Operator means a bounded linear operator.

This paper is motivated by a recent and systematic study of subprojectivity on Banach spaces by T. Oikhberg and E. Spinu [7]. Recall that a Banach space $X$ is called subprojective if any of its subspaces contain a further subspace complemented in $X$.

The simplest examples of subprojective Banach spaces are $\ell_{p}$ spaces $(1 \leq p<\infty)$, $c_{0}$ [9, Lemma 2] and the spaces $C(\alpha)$ of all scalar continuous functions defined on the interval of countable ordinals $[1, \alpha]$, endowed with the order topology [10, Main Theorem]. On the other hand, by Milutin's theorem, [11, Theorem 21.5.10] and [9. Corollary 2], the spaces $C(K)$ of all scalar countinuous functions defined on an uncountable compact metric space are not subprojective.

In their paper, T. Oikhberg and E. Spinu examine, among other things, the stability of the subprojectivity under projective and injective tensor products. Here we turn our attention to the subprojectivity of the projective tensor product $X \widehat{\otimes} Y$ of two subprojective Banach spaces $X$ and $Y$ [7, Question 3.12].

It is known that $\ell_{p} \widehat{\otimes} \ell_{q}, \ell_{p} \widehat{\otimes} c_{0}$ and $c_{0} \widehat{\otimes} c_{0}(1 \leq p, q<\infty)$ are subprojective ([7, Corollary 3.3] and [8, Theorem 5]).

In order to state our main result we need to recall that a Banach space $X$ is said to be $c_{0}$-saturated if any of its subspaces contain a subspace isomorphic to $c_{0}$. The spaces $C(\alpha)$ are also $c_{0}$-saturated for every countable ordinal $\alpha$ [10, Main Theorem].

Received by the editors April 8, 2015 and, in revised form, August 9, 2015.

2010 Mathematics Subject Classification. Primary 46B03; Secondary 46B25.

Key words and phrases. Space of compact operators, spaces of nuclear operators, isomorphic classification, the space $C\left(\omega^{\omega}\right)$. 
In this paper we use a method which is different from that used by OikhbergSpinu [7] and Oja [8]. Here we use Tong's results on diagonal block operators [14] and we show:

Theorem 1.1. Let $K_{1}$ and $K_{2}$ be two countable compact metric spaces. Then $C\left(K_{1}\right) \widehat{\otimes} C\left(K_{2}\right)$ is $c_{0}$-saturated.

So, we deduce easily from the theorem of Sobczyk 13 the "sufficient" part of the following theorem.

Theorem 1.2. Let $K_{1}$ and $K_{2}$ be two compact metric spaces. Then $C\left(K_{1}\right) \widehat{\otimes} C\left(K_{2}\right)$ is subprojective if and only if $K_{1}$ and $K_{2}$ are countable.

Denote by $\omega$ the first infinite ordinal and by $\omega_{1}$ the first uncountable ordinal. By the classical theorem of Mazurkiewicz and Sierpiński [6] we know that an infinite countable compact metric space is homeomorphic to an interval $[1, \alpha]$, where $\omega \leq$ $\alpha<\omega_{1}$.

Thus, we will establish Theorem 1.1 by proving:

Theorem 1.3. Suppose that $\omega \leq \alpha, \beta<\omega_{1}$. Then $C(\alpha) \widehat{\otimes} C(\beta)$ is $c_{0}$-saturated.

\section{Some PRELIMINARY RESUlts}

We start by recalling some basic facts on projective tensor products of Banach spaces [3], [12]. Let $E, F$ be two Banach spaces and denote by $\mathcal{B}(E, F)$ the space of bounded bilinear functionals on $E \times F$. The projective tensor norm of $u=$ $\sum_{i=1}^{n} a_{i} \otimes b_{i} \in E \otimes F$ is defined by

$$
\|u\|=\sup \left\{\left|\sum_{i=1}^{n} \varphi\left(a_{i}, b_{i}\right)\right|: \varphi \in \mathcal{B}(E, F),\|\varphi\| \leq 1\right\} .
$$

As usual, $E \widehat{\otimes} F$ denotes the completion of $E \otimes F$ with respect to the projective tensor norm. If necessary we denote by \|\|$_{\pi(E \otimes F)}$ the projective tensor norm on $E \widehat{\otimes} F$.

We recall that if $M$ is a subspace of $F$, the norm induced by \|\|$_{\pi(E \otimes F)}$ on $E \otimes M$ is not necessarily equivalent to the norm \|\|$_{\pi(E \otimes M)}$. Nevertheless, we have the following result of Grothendieck ([3, Corollary 1, p. 40], [12, Proposition 2.4]) which will play an important role in this work.

Theorem 2.1. Let $E$ and $F$ be Banach spaces. Suppose that $M$ is a complemented subspace of $F$ and $P$ is a bounded linear projection from $F$ onto $M$. Then for every $u \in E \widehat{\otimes} M$, we have $\|u\|_{\pi(E \otimes F)} \leq\|u\|_{\pi(E \otimes M)} \leq\|P\|\|u\|_{\pi(E \otimes F)}$.

We need to introduce the definition of a diagonal block sequence used by Tong in his paper 14 .

Throughout this paper we denote by $\left(P_{m}\right)_{m>1}$ the sequence of natural projections associated to the unit vector basis of $c_{0}$. It is convenient to define $P_{0}=0$.

We say that a sequence $\left(u_{k}\right)_{k \geq 1}$ of $c_{0} \widehat{\otimes} c_{0}$ is a diagonal block sequence if there exist a strictly increasing $\left(n_{k}\right)_{k \geq 1}$ of integers such that $u_{1}=\left(P_{n_{1}} \otimes P_{n_{1}}\right)\left(u_{1}\right)$ and $u_{k}=\left(\left(P_{n_{k}}-P_{n_{k-1}}\right) \otimes\left(P_{n_{k}}-P_{n_{k-1}}\right)\right)\left(u_{k}\right)$ for every integer $k \geq 2$. We prove Theorem 1.3 by a transfinite induction, and the first step is the following lemma.

Lemma 2.2. $c_{0} \widehat{\otimes} c_{0}$ is $c_{0}$-saturated.

Proof. For every integer $n$ we denote $R_{n}=I_{c_{0}} \otimes P_{n}+P_{n} \otimes I_{c_{0}}-P_{n} \otimes P_{n}$. 
We suppose that $c_{0} \widehat{\otimes} c_{0}$ is not $c_{0}$-saturated, so there exists a subspace $E$ of $c_{0} \widehat{\otimes} c_{0}$ which does not contain a subspace isomorphic to $c_{0}$. We shall show that this leads to a contradiction.

Let $0<\varepsilon<1$. We construct by induction a normalized sequence $\left(x_{k}\right)_{k \geq 1}$ of $E$ and a strictly increasing sequence $\left(n_{k}\right)_{k \geq 0}$ of integers such that $n_{0}=0$ which satisfy, for every integer $k \geq 1$,

$$
\left\|\left(\left(P_{n_{k}}-P_{n_{k-1}}\right) \otimes\left(P_{n_{k}}-P_{n_{k-1}}\right)\right)\left(x_{k}\right)-x_{k}\right\| \leq \frac{\varepsilon}{2^{k}} .
$$

To begin we choose $x_{1} \in E,\left\|x_{1}\right\|=1$. We have $x_{1}=\lim _{n \rightarrow \infty}\left(P_{n} \otimes P_{n}\right)\left(x_{1}\right)$ so we can fix an integer $n_{1}$ such that

$$
\left\|x_{1}-\left(P_{n_{1}} \otimes P_{n_{1}}\right)\left(x_{1}\right)\right\| \leq \frac{\varepsilon}{2} .
$$

Now let $i \geq 1$ and suppose that we have a normalized finite sequence $\left(x_{k}\right)_{1 \leq k \leq i}$ of $E$ and a finite sequence of integers $n_{1}<\cdots<n_{i}$ such that (2.1) is satisfied for $1 \leq k \leq i$.

The subspace $\operatorname{Im} R_{n_{i}}$ of $c_{0} \widehat{\otimes} c_{0}$ is isomorphic to $c_{0}$, and so it is $c_{0}$-saturated. It follows that $R_{n_{i}}$ is not an isomorphism from $E$ onto $R_{n_{i}}(E)$, so there exists $x_{i+1} \in E$ which satisfies $\left\|x_{i+1}\right\|=1$ and $\left\|R_{n_{i}}\left(x_{i+1}\right)\right\| \leq \varepsilon / 2^{i+2}$. There exists an integer $n_{i+1}>n_{i}$ such that

$$
\left\|x_{i+1}-\left(P_{n_{i+1}} \otimes P_{n_{i+1}}\right)\left(x_{i+1}\right)\right\| \leq \frac{\varepsilon}{2^{i+2}} .
$$

It is easy to infer from the last inequalities that $x_{i+1}$ satisfies (2.1). Let $z_{k}=$ $\left(P_{n_{k}}-P_{n_{k-1}}\right) \otimes\left(P_{n_{k}}-P_{n_{k-1}}\right)\left(x_{k}\right)$. The sequence $\left(z_{k}\right)_{k}$ is a seminormalized diagonal block sequence of $c_{0} \widehat{\otimes} c_{0}$, so by the result of Tong, [14, Theorem 4.6], it is equivalent to the unit basis of $c_{0}$. For $\varepsilon>0$ small enough, $\left(x_{k}\right)_{k}$ is a basic sequence of $E$ equivalent to the unit basis of $c_{0}$; hence we have a contradiction.

For the continuation it is convenient to introduce new notation. We denote by $C_{0}(\alpha)$ the subspace of $C(\alpha)$ given by $\{f \in C(\alpha) ; f(\alpha)=0\}$. According to [1, Lemma 1] $C_{0}(\alpha)$ is isomorphic to $C(\alpha)$ for $\omega \leq \alpha$. Let $0 \leq \beta<\gamma<\alpha$. We denote this by

$$
C([\beta+1, \gamma])=\left\{f \in C_{0}(\alpha) ; f=f 1_{[\beta+1, \gamma]}\right\},
$$

where $1_{[\beta+1, \gamma]}$ is the characteristic function of the interval $[\beta+1, \gamma]$. Let $1 \leq \gamma \leq \alpha$ be an ordinal. We denote by $S_{\gamma}$ the operator from $C_{0}(\alpha)$ to $C_{0}(\alpha)$ defined by $S_{\gamma}(f)=f 1_{[1, \gamma]}$.

For every $(m, \gamma) \in[1, \omega) \times[1, \alpha)$, we denote by $T_{m, \gamma}$ the operator of $c_{0} \widehat{\otimes} C_{0}(\alpha)$ given by

$$
P_{m} \otimes I_{C_{0}(\alpha)}+I_{c_{0}} \otimes S_{\gamma}-P_{m} \otimes S_{\gamma}
$$

We know [2, Lemma 5.1] that $\operatorname{Im} T_{m, \gamma}$ is isomorphic to $C_{0}(\alpha) \times c_{0} \widehat{\otimes} C(\gamma)$.

The previous lemma is the first step $\alpha=\omega$ of the proof by a transfinite induction of the following lemma.

Lemma 2.3. For every ordinal number $\omega \leq \alpha<\omega_{1}$, the space $c_{0} \widehat{\otimes} C_{0}(\alpha)$ is $c_{0}$ saturated.

Proof. Let $\alpha$ be a countable ordinal such that, for every $\omega \leq \gamma<\alpha$, the space $c_{0} \widehat{\otimes} C_{0}(\gamma)$ is $c_{0}$-saturated. It is obvious that $c_{0} \widehat{\otimes} C_{0}(\alpha)$ is $c_{0}$-saturated if $\alpha$ is a successor. Suppose now that $\alpha$ is a limit ordinal and that $c_{0} \widehat{\otimes} C_{0}(\alpha)$ is not $c_{0^{-}}$ saturated. This means that there exists a subspace $E$ of $c_{0} \widehat{\otimes} C_{0}(\alpha)$ which does not 
contain a subspace isomorphic to $c_{0}$. Let $0<\varepsilon<1$. We construct by induction a normalized sequence $\left(x_{i}\right)_{i \geq 1}$ of $E$, a strictly increasing sequence $\left(m_{i}\right)_{i \geq 1}$ of integers and a strictly increasing sequence $\left(\gamma_{i}\right)_{i \geq 1}$ of $[1, \alpha)$ such that

$$
\left\|x_{1}-\left(P_{m_{1}} \otimes S_{\gamma_{1}}\right)\left(x_{1}\right)\right\| \leq \frac{\varepsilon}{2}
$$

and, for every integer $i \geq 2$,

$$
\left\|x_{i}-\left(\left(P_{m_{i}}-P_{m_{i-1}}\right) \otimes\left(S_{\gamma_{i}}-S_{\gamma_{i-1}}\right)\right)\left(x_{i}\right)\right\| \leq \frac{\varepsilon}{2^{i}} .
$$

We choose $x_{1} \in E,\left\|x_{1}\right\|=1$. We know that $x_{1}=\lim _{m \rightarrow \infty, \gamma \rightarrow \alpha}\left(P_{m} \otimes S_{\gamma}\right)\left(x_{1}\right)$, so we may fix an integer $m_{1}$ and an ordinal $\gamma_{1}<\alpha$ such that (2.2) holds. Let $i$ be an integer $\geq 1$ and suppose we have a finite normalized sequence $\left(x_{k}\right)_{1 \leq k \leq i}$ of $E$, a finite sequence of integers $m_{1}<\cdots<m_{i}$ and a finite sequence $\gamma_{1}<\cdots<\gamma_{i}$ of $[1, \alpha)$ such that (2.2) and (2.3) are satisfied for $k=1, \ldots, i$. It follows from our assumption and from [5, Lemma 1] that $C_{0}(\alpha) \times c_{0} \widehat{\otimes} C(\gamma)$ is $c_{0}$-saturated. Hence $T_{m_{i}, \gamma_{i}}$ is not an isomorphism from $E$ onto its image so there exists $x_{i+1} \in E$ such that $\left\|x_{i+1}\right\|=1$ and

$$
\left\|T_{m_{i}, \gamma_{i}}\left(x_{i+1}\right)\right\| \leq \varepsilon / 2^{i+2} .
$$

There exist an integer $m_{i+1}>m_{i}$ and an ordinal $\gamma_{i}<\gamma_{i+1}<\alpha$ such that

$$
\left\|x_{i+1}-\left(P_{m_{i+1}} \otimes S_{\gamma_{i+1}}\right)\left(x_{i+1}\right)\right\| \leq \varepsilon / 2^{i+2} .
$$

We deduce easily from (2.4) and (2.5) that (2.3) holds for $i+1$. Let $z_{1}=\left(P_{m_{1}} \otimes\right.$ $\left.S_{\gamma_{1}}\right)\left(x_{1}\right)$ and, for $i \geq 2, z_{i}=\left(P_{m_{i}}-P_{m_{i-1}}\right) \otimes\left(S_{\gamma_{i}}-S_{\gamma i_{1}}\right)\left(x_{i}\right)$. The sequence $\left(z_{i}\right)_{i>1}$ is a seminormalized diagonal block sequence of $c_{0} \widehat{\otimes} C_{0}(\alpha)$, and thus, by [2, Theorem 4.2], it is equivalent to the unit basis of $c_{0}$. For $\varepsilon>0$ small enough the sequence $\left(x_{i}\right)_{i \geq 1}$ is a basic sequence of $E$ equivalent to $\left(z_{i}\right)_{i \geq 1}$; hence we have a contradiction.

\section{Diagonal Block Sequences in $C_{0}(\alpha) \widehat{\otimes} C_{0}(\beta)$ SPaCeS}

In order to prove the result announced we introduce diagonal block sequences in $C_{0}(\alpha) \widehat{\otimes} C_{0}(\beta)$. Let $\left(\gamma_{i}\right)_{i \geq 1}$ be a strictly increasing sequence of $[1, \alpha)$ and $\left(\theta_{i}\right)_{i \geq 1}$ a strictly increasing sequence of $[1, \beta)$. We denote by $R_{\theta_{i}}$ the operator of $C_{0}(\beta)$ defined by $R_{\theta_{i}}(g)=g 1_{\left[1, \theta_{i}\right]}$ for every $g \in C_{0}(\beta)$.

Definition 3.1. For every $1 \leq k<\omega$, we denote by $\Pi_{k}$ the operator of $C_{0}(\alpha) \widehat{\otimes} C_{0}(\beta)$ given by

$$
\Pi_{k}(u)= \begin{cases}\left(S_{\gamma_{1}} \otimes R_{\theta_{1}}\right)(u) & \text { if } k=1, \\ \left(\left(S_{\gamma_{k}}-S_{\gamma_{k-1}}\right) \otimes\left(R_{\theta_{k}}-R_{\theta_{k-1}}\right)\right)(u) & \text { if } k \geq 2 .\end{cases}
$$

We say that a sequence $\left(f_{k}\right)_{k \geq 1}$ of $C_{0}(\alpha) \widehat{\otimes} C_{0}(\beta)$ is a diagonal block sequence associated to the sequences $\left(\theta_{k}\right)_{k \geq 1}$ and $\left(\gamma_{k}\right)_{k \geq 1}$ if $\Pi_{k}\left(f_{k}\right)=f_{k}$ for every integer $k$.

Lemma 3.2. Let $\omega \leq \alpha, \beta<\omega_{1}$. Every normalized diagonal block sequence of $C_{0}(\alpha) \widehat{\otimes} C_{0}(\beta)$ is equivalent to the unit basis of $c_{0}$. 
Proof. Let $\left(f_{k}\right)_{k \geq 1}$ be a normalized diagonal block sequence of $C_{0}(\alpha) \widehat{\otimes} C_{0}(\beta)$ associated to the sequences $\left(\theta_{k}\right)_{k \geq 1}$ and $\left(\gamma_{k}\right)_{k \geq 1}$. Let $0<\varepsilon<1$. For every integer $k \geq 1$ we fix $g_{k} \in C_{0}(\alpha) \otimes C_{0}(\beta)$ such that $\left\|f_{k}-g_{k}\right\| \leq \varepsilon / 2^{k}$. We denote $h_{k}=\Pi_{k}\left(g_{k}\right)$; it is obvious that $\left\|f_{k}-h_{k}\right\| \leq \varepsilon / 2^{k}$. For every integer $k$ there exist an integer $n_{k}$ and $u_{1}^{k}, \ldots, u_{n_{k}}^{k} \in C_{0}(\alpha), v_{1}^{k}, \ldots, v_{n_{k}}^{k} \in C_{0}(\beta)$ such that $h_{k}=\sum_{n=1}^{n_{k}} u_{n}^{k} \otimes v_{n}^{k}$. We may suppose that $\left(S_{\gamma_{k}}-S_{\gamma_{k-1}}\right)\left(u_{n}^{k}\right)=u_{n}^{k}$ and $\left(R_{\theta_{k}}-R_{\theta_{k-1}}\right)\left(v_{n}^{k}\right)=v_{n}^{k}$ for every integer $1 \leq n \leq n_{k}$.

The subspace

$$
E_{k}=\left\{u \in C_{0}(\alpha) ; u=\left(S_{\gamma_{k}}-S_{\gamma_{k-1}}\right)(u)\right\}
$$

is a $\mathcal{L}_{\infty, 1+\varepsilon}$ space for every $\varepsilon>0$, so there exists a finite dimensional subspace $X_{k}$ of $E_{k}$ such that $u_{1}^{k}, \ldots, u_{n_{k}}^{k} \in X_{k}, d\left(X_{k}, \ell_{\infty}^{d_{k}}\right) \leq 2$ and a projection $\pi_{k}$ of $E_{k}$ onto $X_{k},\left\|\pi_{k}\right\| \leq 2$, where $d_{k}$ is the dimension of $X_{k}$.

In the same way the subspace

$$
F_{k}=\left\{v \in C_{0}(\beta) ; v=\left(R_{\theta_{k}}-R_{\theta_{k-1}}\right)(v)\right\}
$$

is a $\mathcal{L}_{\infty, 1+\varepsilon}$ space for every $\varepsilon>0$, so there exists a finite dimensional subspace $Y_{k}$ of $F_{k}$ such that $v_{1}^{k}, \ldots, v_{n_{k}}^{k} \in Y_{k}, d\left(Y_{k}, \ell_{\infty}^{d_{k}^{\prime}}\right) \leq 2$ and a projection $\pi_{k}^{\prime}$ of $F_{k}$ onto $Y_{k}$, $\left\|\pi_{k}^{\prime}\right\| \leq 2$, where $d_{k}^{\prime}$ is the dimension of $Y_{k}$.

Let $N$ be an integer. We denote $X=X_{1}+\cdots+X_{N}, Y=Y_{1}+\cdots+Y_{N}, d=$ $d_{1}+\cdots+d_{N}, d^{\prime}=d_{1}^{\prime}+\cdots+d_{N}^{\prime}$. It is clear that $d\left(X, \ell_{\infty}^{d}\right) \leq 2$ and $d\left(Y, \ell_{\infty}^{d^{\prime}}\right) \leq 2$.

Let $\pi$ be the operator from $C_{0}(\alpha)$ onto $X$ defined for every $u$ by

$$
\pi(u)(\gamma)= \begin{cases}\pi_{1} S_{\gamma_{1}}(u)(\gamma) & \text { if } 1 \leq \gamma \leq \gamma_{1}, \\ \pi_{k}\left(S_{\gamma_{k}}-S_{\gamma_{k-1}}\right)(u)(\gamma) & \text { if } \gamma_{k-1}+1 \leq \gamma \leq \gamma_{k} \text { and } 2 \leq k \leq N,\end{cases}
$$

where $\pi$ is a projection and $\|\pi\| \leq 2$.

In the same way the operator $\pi^{\prime}$ from $C_{0}(\beta)$ onto $Y$ defined for every $v$ by

$$
\pi^{\prime}(v)(\gamma)= \begin{cases}\pi_{1}^{\prime} R_{\theta_{1}}(v)(\gamma) & \text { if } 1 \leq \gamma \leq \gamma_{1} \\ \pi_{k}^{\prime}\left(R_{\theta_{k}}-R_{\theta_{k-1}}\right)(v)(\gamma) & \text { if } \gamma_{k-1}+1 \leq \gamma \leq \gamma_{k} \text { and } 2 \leq k \leq N\end{cases}
$$

is a projection and $\left\|\pi^{\prime}\right\| \leq 2$.

The operator $\pi \otimes \pi^{\prime}$ is a projection onto $X \otimes Y$ of norm $\leq 4$.

Let $\lambda_{1}, \ldots, \lambda_{N}$ be scalars; by [3, Corollaire 1, p. 40] we have

$$
\left\|\sum_{i=1}^{N} \lambda_{i} h_{i}\right\|_{\pi\left(C_{0}(\alpha) \otimes C_{0}(\beta)\right)} \leq\left\|\sum_{i=1}^{N} \lambda_{i} h_{i}\right\|_{\pi(X \otimes Y)} \leq 4\left\|\sum_{i=1}^{N} \lambda_{i} h_{i}\right\|_{\pi\left(C_{0}(\alpha) \otimes C_{0}(\beta)\right)} .
$$

Now we use Tong's result to compute $\left\|\sum_{i=1}^{N} \lambda_{i} h_{i}\right\|_{\pi(X \otimes Y)}$.

For every integer $1 \leq i \leq N$, let $T_{i}: X_{i} \rightarrow \ell_{\infty}^{d_{i}}\left(\right.$ resp. $\left.T_{i}^{\prime}: Y_{i} \rightarrow \ell_{\infty}^{d_{i}^{\prime}}\right)$ be an isomorphism such that $\left\|T_{i}\right\|=1$ and $\left\|T_{i}^{-1}\right\| \leq 2$ (resp. $\left\|T_{i}^{\prime}\right\|=1$ and $\left.\left\|T_{i}^{\prime-1}\right\| \leq 2\right)$. The operator $T \otimes T^{\prime}: X \widehat{\otimes} Y \rightarrow \ell_{\infty}^{d} \widehat{\otimes} \ell_{\infty}^{d^{\prime}}$ is an isomorphism such that $\left\|T \otimes T^{\prime}\right\|=1$ and $\left\|T^{-1} \otimes T^{\prime-1}\right\| \leq 4$. We have

$$
\frac{1}{4}\left\|\sum_{i=1}^{N} \lambda_{i} h_{i}\right\|_{\pi(X \otimes Y)} \leq\left\|\sum_{i=1}^{N} \lambda_{i}\left(T \otimes T^{\prime}\right)\left(h_{i}\right)\right\|_{\pi\left(\ell_{\infty}^{d} \otimes \ell_{\infty}^{d^{\prime}}\right)} \leq\left\|\sum_{i=1}^{N} \lambda_{i} h_{i}\right\|_{\pi(X \otimes Y)} .
$$


The sequence $\left(\left(T \otimes T^{\prime}\right)\left(h_{i}\right)\right)_{1 \leq i \leq N}$ is a diagonal block sequence of $\ell_{\infty}^{d} \widehat{\otimes} \ell_{\infty}^{d^{\prime}}$ and so, by Tong's result,

$$
\left\|\sum_{i=1}^{N} \lambda_{i}\left(T \otimes T^{\prime}\right)\left(h_{i}\right)\right\|_{\pi\left(\ell_{\infty}^{d} \otimes \ell_{\infty}^{d^{\prime}}\right)}=\max _{1 \leq i \leq N}\left|\lambda_{i}\right|\left\|\left(T \otimes T^{\prime}\right)\left(h_{i}\right)\right\| .
$$

Hence $\left(h_{i}\right)_{i \geq 1}$ is a seminormalized basic sequence equivalent to the unit sequence of $c_{0}$.

For $\varepsilon>0$ small enough the sequence $\left(f_{i}\right)_{i \geq 1}$ is equivalent to $\left(h_{i}\right)_{i \geq 1}$ and thus equivalent to the unit basis of $c_{0}$.

\section{Proof of the MAIN THEOREM}

We are now ready to prove Theorem 1.3 and the "necessary" part of Theorem 1.2 .

Proof of Theorem 1.3. For every ordinal $\omega \leq \beta<\omega_{1}$ we consider the property

$\left(\mathcal{P}_{\beta}\right) \quad$ for every $\omega \leq \alpha<\omega_{1}$ the space $C(\alpha) \widehat{\otimes} C(\beta)$ is $c_{0}$-saturated.

We show by a transfinite induction that $\left(\mathcal{P}_{\beta}\right)$ is true for every $\beta$. The property $\left(\mathcal{P}_{\omega}\right)$ is true by Lemma 2.2 .

We suppose that $\omega<\beta<\omega_{1}$ is a limit ordinal and that $\left(\mathcal{P}_{\theta}\right)$ is true for every $\omega \leq \theta<\beta$.

Now we show, by a transfinite induction on the ordinal $\alpha \in\left\langle\omega, \omega_{1}\right)$, that $C_{0}(\alpha) \widehat{\otimes}$ $C_{0}(\beta)$ is $c_{0}$-saturated. The result is true if $\alpha=\omega$.

We suppose that $\alpha$ is a limit ordinal and that $C_{0}(\gamma) \widehat{\otimes} C_{0}(\beta)$ is $c_{0}$-saturated for every $\omega \leq \gamma<\alpha$.

We suppose also that $C_{0}(\alpha) \widehat{\otimes} C_{0}(\beta)$ is not $c_{0}$-saturated and show that this assumption leads to a contradiction.

So there exists a subspace $E$ of $C_{0}(\alpha) \widehat{\otimes} C_{0}(\beta)$ which does not contain a subspace isomorphic to $c_{0}$. Let $0<\varepsilon<1$. It is easy to construct by induction a normalized sequence $\left(f_{i}\right)_{i \geq 1}$ of $E$, a strictly increasing sequence $\left(\gamma_{i}\right)_{i \geq 1}$ of $\langle 1, \alpha\rangle$ and a strictly increasing sequence $\left(\theta_{i}\right)_{i \geq 1}$ of $\langle 1, \beta\rangle$ such that

- $\left\|f_{1}-\left(S_{\gamma_{1}} \otimes R_{\theta_{1}}\right)\left(f_{1}\right)\right\| \leq \varepsilon / 2$,

- for every integer $i \geq 2,\left\|f_{i}-\left(\left(S_{\gamma_{i}}-S_{\gamma_{i-1}}\right) \otimes\left(R_{\theta_{i}}-R_{\theta_{i-1}}\right)\right)\left(f_{i}\right)\right\| \leq \varepsilon / 2^{i}$.

It follows by Lemma 3.2 that for $\varepsilon>0$ small enough the sequence $\left(f_{i}\right)_{i \geq 1}$ is equivalent to the unit basis of $c_{0}$ in contradiction with our assumption.

Proof of the "necessary" part of Theorem 1.2. Let $K_{1}$ and $K_{2}$ be two infinite compact metric spaces and suppose that $C\left(K_{1}\right) \widehat{\otimes} C\left(K_{2}\right)$ is subprojective. It follows that $C\left(K_{1}\right) \widehat{\otimes} c_{0}$ is subprojective, so by [7, Proposition 4.2.] the compact $K_{1}$ is scattered.

\section{REFERENCES}

[1] C. Bessaga and A. Pełczyński, Spaces of continuous functions. IV. On isomorphical classification of spaces of continuous functions, Studia Math. 19 (1960), 53-62. MR0113132 (22 \#3971)

[2] Elói Medina Galego and Christian Samuel, The classical subspaces of the projective tensor products of $\ell_{p}$ and $C(\alpha)$ spaces, $\alpha<\omega_{1}$, Studia Math. 214 (2013), no. 3, 237-250, DOI 10.4064/sm214-3-3. MR3061511

[3] Alexandre Grothendieck, Produits tensoriels topologiques et espaces nucléaires (French), Mem. Amer. Math. Soc. 1955 (1955), no. 16, 140. MR0075539 (17,763c) 
[4] William B. Johnson and Joram Lindenstrauss, Basic concepts in the geometry of Banach spaces, Handbook of the geometry of Banach spaces, Vol. I, North-Holland, Amsterdam, 2001, pp. 1-84, DOI 10.1016/S1874-5849(01)80003-6. MR.1863689 (2003f:46013)

[5] Denny H. Leung, Some stability properties of $c_{0}$-saturated spaces, Math. Proc. Cambridge Philos. Soc. 118 (1995), no. 2, 287-301, DOI 10.1017/S0305004100073643. MR.1341791 (97e:46015)

[6] S. Mazurkiewicz and W. Sierpiński, Contributions à la topologie des ensembles dénombrables, Fund. Math. 1 (1920), 513-522.

[7] T. Oikhberg and E. Spinu, Subprojective Banach spaces, J. Math. Anal. Appl. 424 (2015), no. 1, 613-635, DOI 10.1016/j.jmaa.2014.11.008. MR3286583

[8] Eve Oja, Sur la réflexivité des produits tensoriels et les sous-espaces des produits tensoriels projectifs (French), Math. Scand. 51 (1982), no. 2, 275-288 (1983). MR690532 (84i:46071)

[9] A. Pełczyński, Projections in certain Banach spaces, Studia Math. 19 (1960), 209-228. MR0126145 (23 \#A3441)

[10] A. Pełczyński and Z. Semadeni, Spaces of continuous functions. III. Spaces $C(\Omega)$ for $\Omega$ without perfect subsets, Studia Math. 18 (1959), 211-222. MR0107806 (21 \#6528)

[11] Haskell P. Rosenthal, The Banach spaces $C(K)$, Handbook of the geometry of Banach spaces, Vol. 2, North-Holland, Amsterdam, 2003, pp. 1547-1602, DOI 10.1016/S1874-5849(03)800438. MR1999603 (2004g:46028)

[12] Raymond A. Ryan, Introduction to tensor products of Banach spaces, Springer Monographs in Mathematics, Springer-Verlag London, Ltd., London, 2002. MR.1888309 (2003f:46030)

[13] Andrew Sobczyk, Projection of the space $(m)$ on its subspace $\left(c_{0}\right)$, Bull. Amer. Math. Soc. 47 (1941), 938-947. MR0005777 (3,205f)

[14] Alfred Tong, Diagonal nuclear operators on $l_{p}$ spaces, Trans. Amer. Math. Soc. 143 (1969), 235-247. MR0251559 (40 \#4786)

Department of Mathematics, University of São Paulo, SÃo Paulo, Brazil 05508-090

E-mail address: eloi@ime.usp.br

I2M, Aix Marseille Université, CNRS, UMR 7353, 13453 Marseille Cedex 20, France E-mail address: christian.samuel@univ-amu.fr 